\title{
Signals and circuits in the Purkinje neuron
}

\author{
Ze'ev R. Abrams $^{1,2}$ and Xiang Zhang ${ }^{1,2 *}$ \\ ' Applied Science and Technology, Graduate Program University of California Berkeley, Berkeley, CA, USA \\ ${ }^{2}$ NSF Nano-scale Science and Engineering Center, University of California Berkeley, Berkeley, CA, USA
}

\section{Edited by:}

Miles A. Whittington, Newcastle

University, UK

Reviewed by:

Audrey Mercer, University of London, UK

Michael Nitabach, Yale University

School of Medicine, USA

${ }^{*}$ Correspondence:

Xiang Zhang, NSF Nano-scale

Science and Engineering Center,

University of California Berkeley,

Etcheverry Hall, Room 3112, Berkeley,

CA 94720-1740, USA.

e-mail: xiang@berkeley.edu
Purkinje neurons (PN) in the cerebellum have over 100,000 inputs organized in an orthogonal geometry, and a single output channel. As the sole output of the cerebellar cortex layer, their complex firing pattern has been associated with motor control and learning. As such they have been extensively modeled and measured using tools ranging from electrophysiology and neuroanatomy, to dynamic systems and artificial intelligence methods. However, there is an alternative approach to analyze and describe the neuronal output of these cells using concepts from electrical engineering, particularly signal processing and digital/analog circuits. By viewing the PN as an unknown circuit to be reverse-engineered, we can use the tools that provide the foundations of today's integrated circuits and communication systems to analyze the Purkinje system at the circuit level. We use Fourier transforms to analyze and isolate the inherent frequency modes in the PN and define three unique frequency ranges associated with the cells' output. Comparing the PN to a signal generator that can be externally modulated adds an entire level of complexity to the functional role of these neurons both in terms of data analysis and information processing, relying on Fourier analysis methods in place of statistical ones. We also re-describe some of the recent literature in the field, using the nomenclature of signal processing. Furthermore, by comparing the experimental data of the past decade with basic electronic circuitry, we can resolve the outstanding controversy in the field, by recognizing that the PN can act as a multivibrator circuit.

\section{REVERSE-ENGINEERING THE PURKINJE NEURON}

With more neurons than any other region in the brain, and a geometrical architecture that bears a resemblance to a man-made computer, the cerebellum has been the source of numerous theories of motor control (Eccles et al., 1967; Raymond et al., 1996; Doya et al., 2001; Ito, 2006) as well as fundamental concepts in artificial intelligence (Marr, 1969; Albus, 1971; Brunel et al., 2004; Dean et al., 2010). At the heart of this neuronal circuit lies one of the largest types of neuron in the brain: the Purkinje neuron (PN); each with over 100,000 inputs from perpendicular parallel fibers (PFs) and inhibitory interneurons, and a single climbing-fiber (CF) input per cell ascending from the inferior olive (IO; Ito, 2006). The vast complexity of intermingled circuitry, abundance of neurotransmitters and combination of different subtypes of neighboring cells (Ito, 2001) confound efforts of reverse-engineering the PN.

The majority of studies of PN functionality have been centered on spike timing and statistical analyses. At the cellular level, focusing on the neurotransmitters and their dynamics has lead to discoveries such as long-term depression (LTD) at the PF-PN and CF-PN synapses (Ekerot and Kano, 1985; Kano and Kato, 1987; Hansel and Linden, 2000) as well as relating LTD to motor learning (Raymond et al., 1996; Ito, 2001; Dean et al., 2010). At the circuit level, focusing on the orthogonal geometry of the cerebellar cortex has lead to theories of the morphological significance of this architecture in terms of motor function (Rokni et al., 2008; Apps and Hawkes, 2009; Bower, 2010). At this level of description, the most prominent controversy in the field of the $\mathrm{PN}$ is the existence of "Bistability" (as will be defined below) and whether it exists in the natural state (Rokni et al., 2009). The descriptions of Bistability have used spike timing analysis (Loewenstein et al., 2005; De Schutter and Steuber, 2009), which will be shown here to provide only a partial perspective of the controversy.

There is an alternative approach to analyzing and deciphering the functions of the PN, emanating from the field of electrical engineering (EE), particularly in terms of signal processing and circuit analysis. The terms "signal" and "circuit" have particular meanings in EE that are not always carried over when used in the field of neuroscience. The most elementary methodology to reverse engineer the $\mathrm{PN}$, whose functions are unknown, is to treat it as a "black box," and measure the response of the cell (via the axon) as a function of its inputs. In this sense, the input signal must be subdivided into a zero input response - the natural firing pattern of the PN in the absence of inputs; the first-order input response - considering the output response to a variety of external sources; and higher-order responses including feedback and feedforward control mechanisms, where the inputs signals are re-fed into the cell. Distinguishing between these levels of complexity are systembased arguments, where the circuitry plays an important role in shaping the signal. In our paper (Abrams et al., 2010), we measured the output signal of the $\mathrm{PN}$ in in vitro conditions, which are better suited for measuring zero- and first-order responses. Comparisons of in vitro and in vivo analyses must take into account the different circuits involved, with most zero- and first-order results 
unobtainable using the in vivo method, due to the complexity of mixed signals. Furthermore, in terms of signals, the output of a cell can be viewed on a "pulse in, pulse out" level [akin to a direct current (DC) circuit] or on a "signal in, signal out" level [akin to an alternating current (AC) circuit]. The former considers each action potential (AP) to be the significant source of information. The latter of these descriptions deals with trains of APs and is far more powerful, encompassing the former; it relies on concepts of signal processing and particularly, the frequency response of a system.

Previous studies have shown that the PN is a constantly firing cell, even without external inputs (Llinás and Sugimori, 1980; Lang et al., 1999). Other studies of the cerebellum include theories of pattern generation circuits (Yarom and Cohen, 2002; Jacobson et al., 2008) based on oscillations measured in the cerebellum (Maex and De Schutter, 2005; De Zeeuw et al., 2008); however, earlier studies had shown an apparent lack of oscillatory activity in the PN itself (Keating and Thach, 1997; Hakimian et al., 2008). In our paper (Abrams et al., 2010) we showed a clearly measurable oscillatory signal in the $\mathrm{PN}$, and delineated three-frequency bands that we claim are intrinsic to the PN. This review focuses on the results of our paper, and describes an alternative way of viewing $\mathrm{PN}$ functionality, in terms of frequency responses. We describe how using frequency analysis tools provide additional information regarding the output signal of the $\mathrm{PN}$, as well as a brief review of recent results that bolster this approach. Finally, we use a circuitbased approach to show that the primary controversy in the field can be simplified in an elegant fashion, and describe the $\mathrm{PN}$ as a timing circuit.

\section{HARMONIC SIGNALS AND THE FREOUENCY DOMAIN}

The field of signal processing relies on the distinction between the time and frequency domain descriptions of a harmonic signal. Harmonic signals and systems are an indispensible aspect of the natural world, ranging from the crystalline atomic structure of most materials, to the voice patterns we use every day. However, since we encounter most objects on an individual basis, and with a one-directional view of time, the time-domain description is what is normally used to describe things. For example, a single sin wave $[\sin (t)]$ is the most basic form of harmonic signal (Figure 1A, upper left, black), continuing from $-\infty$ to $+\infty$ in time, with an amplitude, $A$. Since it is an infinitely long signal, it would theoretically take infinite space to draw it in the time-domain with horizontal axis $t$. However, there are actually only two quantities of information needed to describe the signal: the amplitude, $A$, and the frequency, $f$, here at $1 \mathrm{~Hz}$. Mathematically, there is a oneto-one transformation between functions in the time-domain and frequency domain: the Fourier transform (FT), which takes any function of $t$ and transforms it into a function of $f$. The FT of the function $\sin (t)$ is a single peak at $1 \mathrm{~Hz}$ (Figure 1A, upper right), signifying that only $1 \mathrm{~b}$ of information (excluding amplitude) is needed to describe this signal. Intuitively, this is obvious in the case of $\sin (t)$ since it is understood from " $\sin (t)$ " that the signal is infinite with a frequency of $1 \mathrm{~Hz}$. A more interesting example is combining $\sin (t), \sin (3 t)$, and $\sin (5 t)$; the frequency domain description is simply three isolated peaks (Figure 1A, blue right), however the time-domain description of this combined signal is
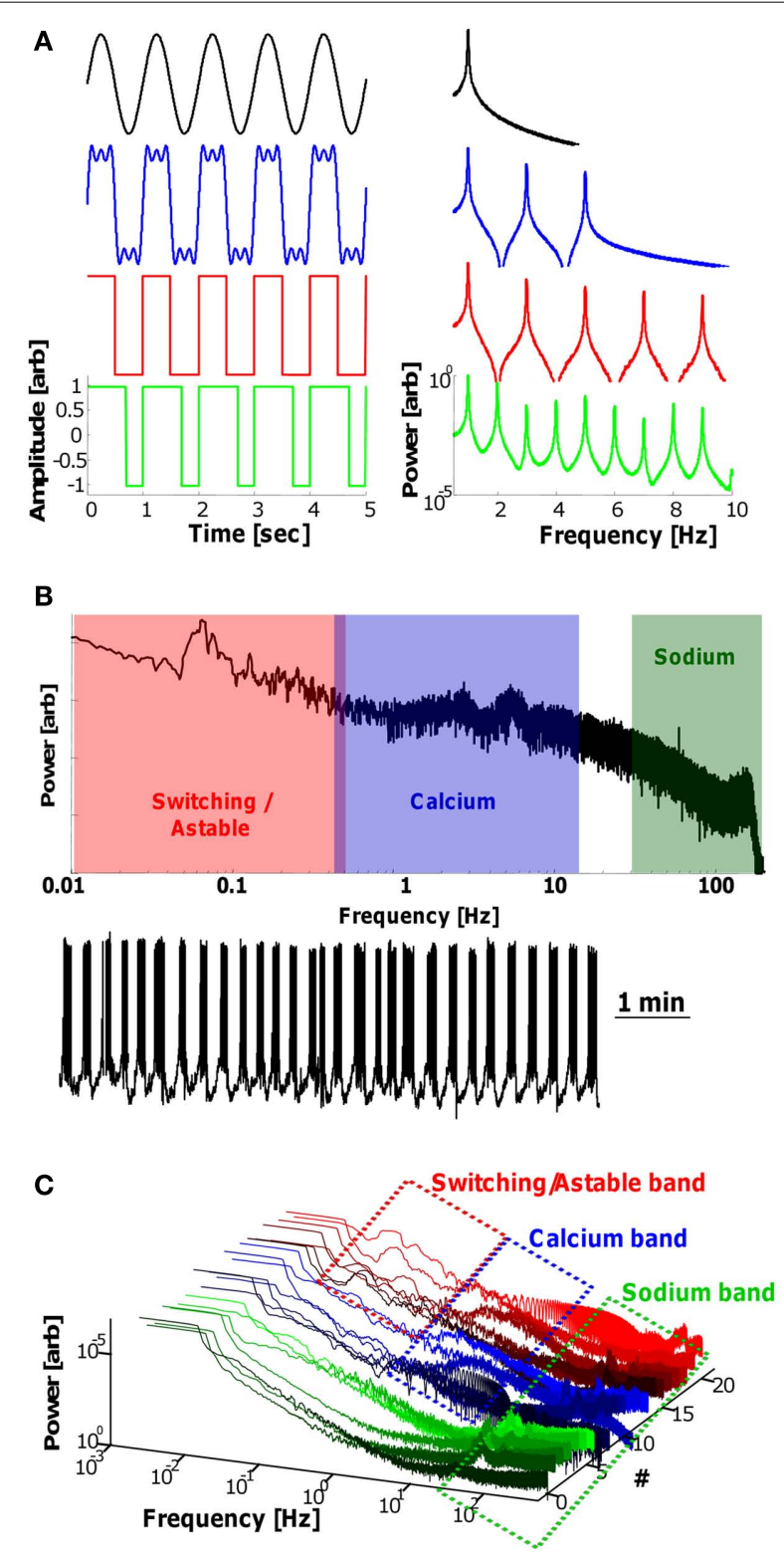

FIGURE 1 | Fourier transforms and Purkinje firing output. (A)

Time-domain (left) and frequency domain (right, logarithmic $y$-axis) descriptions of mathematical signals. From top: (black) a single $1 \mathrm{~Hz}$ sine; (blue) combination of $\sin (t), \sin (3 t)$, and $\sin (5 t)$, approximating a $1 \mathrm{~Hz}$ square-wave; (red) a perfect square-wave can be described as a series of odd integer harmonics of sine waves; (green) a $70 \%$ duty-cycle, $1 \mathrm{~Hz}$ square-wave has integer harmonics with different frequency coefficient heights. (B) FT of a 9 min patch-clamp recording from a Purkinje neuron in vitro (below) consisting of three-frequency range windows: Switching/Astable $(<1 \mathrm{~Hz}$, red); Calcium $(0.7-15 \mathrm{~Hz}$, blue); Sodium $(>30 \mathrm{~Hz}$, green). (C) Twenty-one FTs of 7 min recordings of spontaneously firing Purkinjes in vitro arranged showing all the cells displaying Sodium frequencies, 2/3 with Calcium frequencies, and 1/3 with Switching/Astable frequencies. (see Abrams et al., 2010, for experimental details.).

quite unintuitive, appearing as nearly a square-wave (Figure 1A, blue left). In fact, a perfect square-wave $(1 \mathrm{~Hz})$ can be described as a series of odd integer harmonics of sin waves with different 
amplitudes (Figure 1A, red). If the duty-cycle of the square-wave (duty-cycle is the time "up" over the full period) is altered, the frequency domain description has all-integer components at differing amplitudes (Figure 1A, green). An important observation to be made is that the axes of FT plots are typically logarithmic, to accentuate the peaks, however, this leads to an artificial increase in the "noise" signal, leading to the curved bottoms of the FT plots.

Engineers will typically use the term "frequency" interchangeably with "period" $(f=1 / T)$. Moreover, "frequency" can be used to describe the exact instantaneous frequency of a signal, or the frequency band being described. As an example, when listening to a radio station at $100 \mathrm{MHz}$, the "station" is the frequency band (in this case, $100 \mathrm{MHz}$ ), although the instantaneous "frequency" is continuously modulated between $\sim 20$ and $20,000 \mathrm{~Hz}$ (audible sounds) within this band. Amplitude and frequency modulated (AM/FM) stations rely on different modulation techniques, however both methods will result in a distinct signature in the FT: the region of the "envelope" frequency (viz. $100 \mathrm{MHz}$ ) will have side-bands (i.e., peaks offset to the left and right), which will shift according to the "carrier" signal ( $v i z$. the music being played).

Measuring the output signal of numerous PNs in vitro, we have described three characteristic frequency bands: the Sodium frequency $(>30 \mathrm{~Hz})$, Calcium frequency $(\sim 1-15 \mathrm{~Hz})$ and a newly defined "Switching" frequency $(<1 \mathrm{~Hz})$. The names of the Sodium and Calcium frequency bands are derived from the well-documented spikes that form their respective waveforms: The high-frequency AP $\mathrm{Na}^{+}$spikes exist in every cell, and form the carrier signal that is eventually transmitted as APs downstream (Monsivais et al., 2005). The lower frequency $\mathrm{Ca}^{2+}$ spikes are a more unique attribute of the PN (Hartmann and Konnerth, 2005; Achard and De Schutter, 2008), and are shorter, slower envelope waveforms. The Switching frequency is slower than the signals typically studied by most researchers (occasionally $40+s$ periods), and can only be seen when viewing long time periods (see also Womack and Khodakhah, 2002, 2004; McKay et al., 2007; Chen et al., 2009). Since certain cells display slow, continual oscillations (especially when pharmaceutically induced to do so; see Abrams et al., 2010 for details), we can also call this an "Astable" mode, since it has no stable state, and oscillates between firing and quiescence. The FT of a cell with all three-frequency bands appears in Figure 1B, with the frequency bands overlaid for clarity. The naturally noisy $\mathrm{PN}$ signal has a $1 / f$ fall in amplitude as is typical in naturally occurring signals (Roopun et al., 2008). Seeing the sub- $1 \mathrm{~Hz}$ peaks in the FT requires very long segments of signal to be analyzed, as a rule requiring 10 full oscillation periods to occur within the analysis window. Furthermore, the amplitude of a peak in the FT not only incorporates information of the harmonics of the waveform (as in Figure 1A), but also the duration of time that a certain frequency appears; for example, if a cell fires at $10 \mathrm{~Hz}$ for $10 \mathrm{~s}$ during a $1 \mathrm{~min}$ recording, the peak will be smaller than if it fires at that rate for the entire minute. Therefore, the appearance of a peak in the FT signal signifies the existence of a frequency in the signal, with no information on the location of that frequency in the time-domain.

The FTs of 21 PNs measured spontaneously in vitro (with no additional pharmaceuticals added, $7 \mathrm{~min}$ recordings each) is presented in Figure 1C, arranged such that $1 / 3$ contain
Switching/Astable frequencies, $2 / 3$ contain Calcium frequencies, and all contain Sodium frequencies. The three-frequency bands described here are different from the ambiguously classified " $\gamma$, $\delta, \theta$, etc., frequency bands used in electroencephalography (EEG) measurements, as well as those use in other regions of the brain (Buzsáki and Draguhn, 2004; Steriade, 2006; De Zeeuw et al., 2008; Roopun et al., 2008; Colgin et al., 2009), which is why they are not compared here to these known frequency bands. The names of these bands were given on the basis of the known contributions of their ionic components, particularly $\mathrm{Na}^{+} \mathrm{APs}$ and $\mathrm{Ca}^{2+}$ spikes, which were shown to operate at slightly different frequency ranges from EEG-related bands. Other names can be given to these bands to remove the possible ambiguity between the $\mathrm{Na}^{+} / \mathrm{Ca}^{2+}$ spikes themselves and the frequency bands.

\section{TOOLS OF THE TRADE: ANALYZING COMPLEX OUTPUTS}

To demonstrate the power and utility of the combination of both time and frequency domain representations of the PN output signal, we utilize some signal processing techniques on a segment of PN signal recording: a 20 s segment of recording is displayed and analyzed in Figure 2 (segment was taken at random, from a current-clamp recording of a $\mathrm{PN}$ induced into firing $\mathrm{Ca}^{2+}$ spikes using a kainate receptor agonist; see Abrams et al., 2010 for full experimental details). The full $20 \mathrm{~s}$ signal in Figure 2A consists of a combination of $\mathrm{Na}^{+}$spikes riding on top of $\mathrm{Ca}^{2+}$ spikes, as is best seen in the $200 \mathrm{~ms}$ close-up of a single $\mathrm{Ca}^{2+}$ pulse in Figure 2C. As can be seen from the magnified single pulse in Figure $2 \mathrm{C}$, the APs increase in frequency as a function of the amplitude of the underlying $\mathrm{Ca}^{2+}$ spike; this is known as a chirped signal, and is a demonstration of FM. The "traditional" way to analyze the output segment of Figure 2A would be to plot the autocorrelation and interspike interval (ISI) of the spikes, as is plotted in Figures 2D,E. From the ISI, the typical information inferred are the mean ISI, in this case $8.4 \mathrm{~ms}$, which provides the reciprocal of the frequency, $118 \mathrm{~Hz}$, and the standard deviation (SD) and coefficient of variation (CV) of the histogram (assuming a Gaussian distribution, which is appropriate for large numbers of pulses). The CV is typically denoted by its inverse in engineering terms, as the signal-to-noise ratio $(\mathrm{SNR}=1 / \mathrm{CV}=\mathrm{Mean} / \mathrm{SD})$. Note that in this ISI plot, there are actually two peaks (at 2.7 and $6.7 \mathrm{~ms}$, marked by a red circle and magenta triangle, respectively), which would initially lead to the conclusion that a double-Gaussian fit must be used to describe the signal; however, as is seen in the time-domain signal of Figure 2C, neither of these frequencies actually appear consistently anywhere in the signal. Furthermore, these two analysis methods lose all lower frequency information, with little to no additional information being extracted from the autocorrelogram (Figure 2D).

In contrast, evaluating the full FT of the signal in Figure 2A, as portrayed in Figure $2 \mathrm{~F}$ (note the logarithmic $x$-axis), provides insight in the frequency domain, where peaks at $\sim 8$ and $\sim 10 \mathrm{~Hz}$ (and their harmonics) are distinct. The high-frequency range (inset, Figure 2F) displays multiple (noisy) peaks at both the $\sim 150$ and $\sim 370 \mathrm{~Hz}$ regions. From the FT, the existence of lowfrequency peaks immediately signifies the existence of another frequency band that is important other than the high-frequency AP spikes, and the "shape" of the high-frequency band indicates a form of modulation AM/FM. 

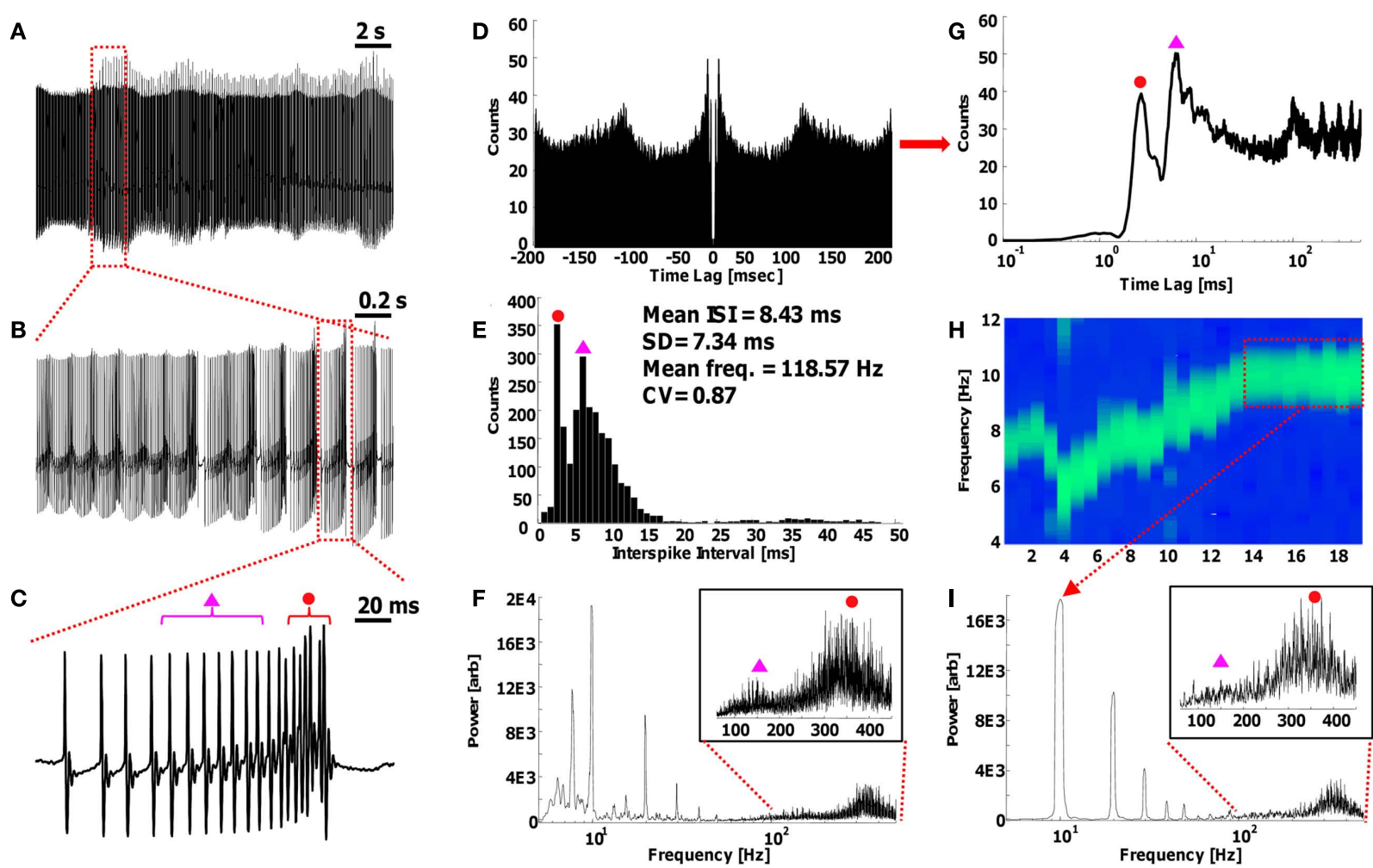

FIGURE 2 | Comparing analysis methods on a segment of Purkinje output signal. (A) Twenty seconds recording of a Purkinje neuron induced into firing $\mathrm{Ca}^{2+}$ spikes (Abrams et al., 2010). (B) Close-up of a $2 \mathrm{~s}$ frequency transition period. (C) Close-up of a single $\mathrm{Ca}^{2+}$ spike (small underlying upward curve), with $\mathrm{Na}^{+}$spikes riding on top (large action potential spikes). The $\mathrm{Na}^{+}$spikes are frequency modulated - chirped - their frequency goes up with the amplitude of the $\mathrm{Ca}^{2+}$ spike. (D) Standard autocorrelogram, and (E) The interspike interval (ISI) of the full $20 \mathrm{~s}$ signal in (A), as traditionally displayed. The autocorrelogram in this form only provides minimal information of the shortest spike times, being the peaks near time lag $=0$. The ISI displays two peaks, with a mean of $8.4 \mathrm{~ms}$ $(=118 \mathrm{~Hz})$. The individual peaks are at $2.7 \mathrm{~ms}$ (red dot) and $6.7 \mathrm{~ms}$ (magenta triangle). The $\mathrm{CV}$ is the noise (SD) over the mean, the reciprocal of the signal-to-noise ratio (SNR). (F) Full FT of (A) low-frequency $\mathrm{Ca}^{2+}$ spikes at $\sim 10 \mathrm{~Hz}$ and very high-frequency $\mathrm{Na}^{+}$spikes at $80-400 \mathrm{~Hz}$. Inset: the
Sodium frequency range, with multiple, noisy peaks (red dot at $370 \mathrm{~Hz}$, magenta triangle at $150 \mathrm{~Hz}$ ). More information of the signal is found here than in (D) and (E), with the multiple peaks in the Calcium and Sodium ranges signifying a complex modulated signal in the time-domain. (G) Semi-logarithmic autocorrelogram of (D), displaying similar information as the ISI in (E). Peaks on the far right are residuals of lower frequency information that are hard to measure using an autocorrelogram. (H) Spectrogram of the Calcium range of the $20 \mathrm{~s}$ signal, which displays localized FTs over time, thus combining the frequency and time-domain information. The $\mathrm{Ca}^{2+}$ spikes shift in frequency over time. (I) Close-up of the full FT for only $4 \mathrm{~s}$ of the signal [red box in $\mathbf{( H )}$ ], with a clear $10 \mathrm{~Hz}$ Calcium frequency peak. Inset: clearer Sodium frequency region spectra. Note that there are no $2.7 / 6.7 \mathrm{~ms}(370 / 150 \mathrm{~Hz})$ spikes in the actual signal (red dot, magenta triangle), and the $\sim 370 \mathrm{~Hz}$ Sodium frequency is modulated by the Calcium frequency $(10 \mathrm{~Hz}$ spacing between peaks).
More information about the signal is nevertheless obtainable by utilizing the correct tools in both the time and frequency domains. Taking the autocorrelogram of Figure 2D, which is by definition symmetric about time lag zero, and only plotting the positive side (which is what is done for the FT since there is no significance to negative frequencies), in logarithmic scale as in Figure 2G, we can obtain the same information as the ISI plot of Figure 2E, demonstrating the "redundancy" of the both plots. To obtain both time and frequency domain information simultaneously, a spectrogram can be plotted of a specific frequency band as in Figure $\mathbf{2 H}$ (see Abrams et al., 2010 for limitations on the use of the spectrogram). The spectrogram displays the variations in the Calcium frequency band over time as peaks in the localized FT, and shows the $\mathrm{Ca}^{2+}$ spikes fluctuating in frequency over time, from 5 to $10 \mathrm{~Hz}$, within the Calcium frequency band. In Abrams et al. (2010), we showed that the Calcium band can also undergo drastic variations such as sudden frequency doubling, as well as a stabilization of the frequency with an RC-like time constant. Focusing on the last $4 \mathrm{~s}$ of the recording, where the Calcium frequency is stabilized (red box in Figure 2H), we can examine the FT of this region (averaged over the $4 \mathrm{~s}$ ) in Figure 2I. Here, the clear $10 \mathrm{~Hz}$ Calcium frequency is apparent, as well as its harmonics (at 20, 30, etc., Hz). The inset of the Sodium frequency band displays a clearer FM modulation centered on 150 and $370 \mathrm{~Hz}$, with sharp internal peaks with $10 \mathrm{~Hz}$ displacements (the side-bands; see Figure 3 in Abrams et al., 2010 for more). Note once again that the $150 / 370 \mathrm{~Hz}(6.7 / 2.7 \mathrm{~ms})$ signal does not exist per se in the time-domain signal, demonstrating the difficulty with using the ISI information alone. Finally, while the curvature of the $\mathrm{Ca}^{2+}$ spikes is quite apparent in the time-domain of the signal (Figures $2 \mathrm{~A}-\mathrm{C}$ ), they would not be visible in many in vivo recordings since external patches effectively act as a low pass filter on the signal (or as a differential, which is equivalent in the 


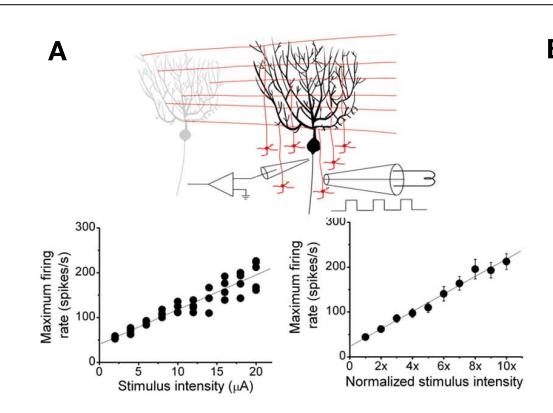

D

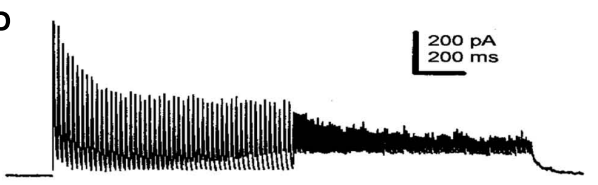

\begin{tabular}{l}
$100 \mathrm{pA}$ \\
\hline $50 \mathrm{~ms}$ \\
\hline
\end{tabular}

ANAIANA MMMMMMMMMMMMan
B
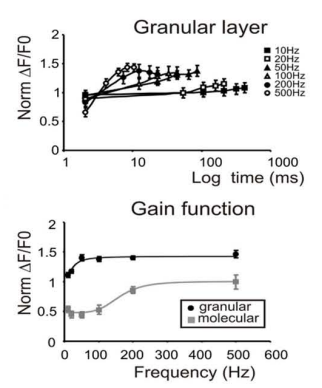

E

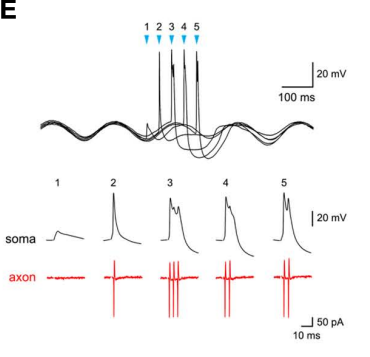

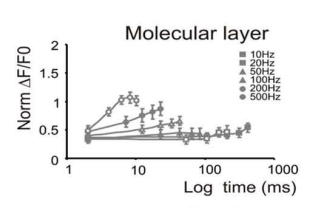

Lag function

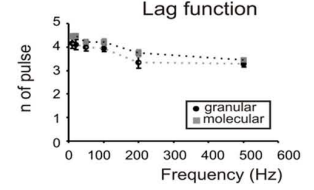

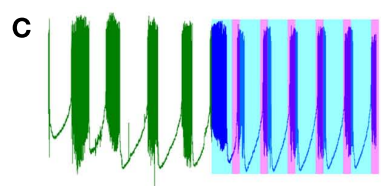

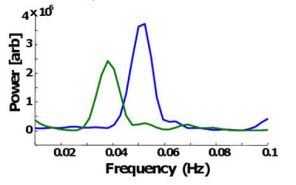

$\mathbf{F}$
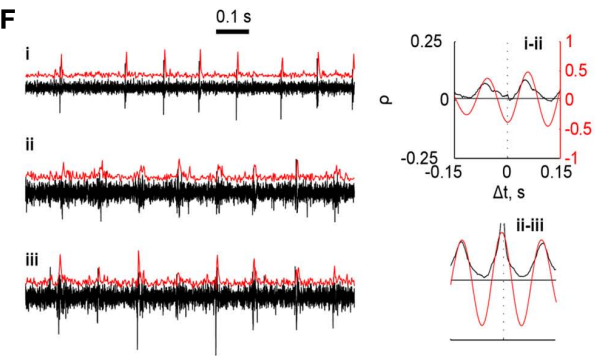

FIGURE 3 | Modulation into, and out of, the Purkinje neuron. (A) Linear output as a function of input: the frequency of Purkinje output firing rises linearly with increased input stimulus (parallel fiber, granule layer) intensity Malter and Khodakhah, 2006). (B) High-pass filtering function of the granule layer (mossy fiber) inputs to the granule layer, measured via fluorescence $(\Delta F / F 0)$. The mossy fibers have a preference of transmitting higher frequency signals (Mapelli et al., 2010). (C) Photo-switchable kainate modulation of the Astability from a naturally oscillating Purkinje (green, left, at $0.04 \mathrm{~Hz}$ ), externally modulated to fire at $0.05 \mathrm{~Hz}(25 \mathrm{~s}$ period, $25 \%$ duty-cycle, unpublished results). (D) Modulation of inhibition of the cerebellar nuclei (artificial stimulation). At higher frequencies of inhibition (from 50 to $100 \mathrm{~Hz}$ ), the efferent cells to the Purkinje are depressed more (Telgkamp and Raman, 2002). (E) Phase-locked modulation of olivary output to sub-threshold oscillations. The firing increases at the peak of the underlying oscillation (frequency modulation), which provides the climbing-fiber input to the Purkinje neuron (Mathy et al., 2009). (F) Phase locking of different Purkinje neurons inhibiting a cerebellar neuron. The top two traces show synchrony between spikes (correlation measured on right), whereas the bottom two traces display little correlation. This form of phase locking can act as an edge detector for the cerebellar nuclei (Jacobson et al., 2009). frequency domain), but this information would still be available in the FT of the signal since the FT is an objective mathematical technique that can isolate frequency peaks that are indistinguishable to the eye in the time-domain. The FT is also an inherently "statistical" process, with the full width at half maximum (FWHM) of a peak being directly related to the $\mathrm{SD}$, thereby enabling a direct measurement of the SNR (and CV) from the spectral peaks.

\section{MODULATION CAPABILITIES OF THE PURKINJE NEURON}

Using the terminology described in the previous sections, we can approach the existing literature on PN firing sequences from the perspective of signal processing. Considering the three frequencies found in the PN output as intrinsic to these cells, a first-order description of the PN output can be described in terms of interacting function generators: the full circuit of the PN is known to include many input contributions (Ito, 2006), which modulate the output of the PN (Häusser and Clark, 1997; Mittmann and Häusser, 2007; Oldfield et al., 2010) as well as the complex contribution of the multitude of excitatory PF inputs (Jaeger and Bower, 1994; Isope and Barbour, 2002; D’Angelo et al., 2009; Mapelli et al., 2010), and the solitary CF excitatory synapse.

A fundamental question that can be asked is whether these modulation concepts (AM/FM) both into and out of the PN can be interpreted by the other neurons in the cerebellum? The experimental evidence of the past few years have garnered quite a few examples demonstrating that this is indeed the case. Using the three-frequency description of the PN, the response of the PN to its inputs (i.e., the frequency response), as well as the modulation effect on its output, is here discussed in relation to each frequency band associated with the modulation; as well as referring to either the input end of the PN, or the output end.

On the input end, the Sodium frequency regime has been the primary focus of most studies of PN signaling. For example, increasing the PF inputs has been shown to have a linear effect on the Sodium frequency (Walter and Khodakhah, 2006), as shown in Figure 3A, demonstrating the most basic form of linear computation and modulation. The granule layer itself (the source of the PF inputs to the PN) has been shown to have high-pass filtering process capabilities in the range of $50+\mathrm{Hz}$ (Mapelli et al., 2010), as shown in Figure 3B, as well as oscillatory behavior (D'Angelo et al., 2009). Furthermore, the contributions of excitatory and inhibitory synapses on the PN have been shown to have a correlated modulatory effect on the firing rate of the PNs (Häusser and Clark, 1997), raising and lowering the Sodium firing rate. We have also shown some modulation of the Calcium and Switching frequencies using kainate agonists (Abrams et al., 2010), including direct modulation of oscillations (Astability) using photo-switching compounds. Recently, we have demonstrated direct modulation of the Switching frequency alone (Figure 3C, unpublished results).

The output end of the PN has likewise been shown to have frequency responses to modulations in input frequencies. These 
include the ability of the deep cerebellar neurons (DCNs, the output cell in the PN circuit) to interpret PN firing rates (Telgkamp and Raman, 2002), shown in Figure 3D, as well as PN output patterns (Baumel et al., 2009). There has also been an increase in studies of AM signaling in the brain (Kramer et al., 2008; Atallah and Scanziani, 2009), with the IO (generator of the CF) being AM modulated to sub-threshold oscillations (Mathy et al., 2009), as shown in Figure 3E. This new emphasis in analysis meshes with our results of a combined frequency output emanating from the $\mathrm{PN}$, which includes AM/FM modulation. Measurements of synchrony between PNs are likewise a way of modulating the DCN output (Wise et al., 2010). In particular, the synchronous activity of PNs on a DCN can act as an edge detector allowing a high range of frequencies to be detected by the DCN (Jacobson et al., 2009), as displayed in Figure 3F. Combinatory frequency responses caused by the occurrence of different frequency outputs have also been seen in other systems (Geisler et al., 2010), with the ability of individual cells such as the $\mathrm{PN}$ to produce a wide variety of frequencies being an integral element of theories of cerebellar temporal pattern generation (Yarom and Cohen, 2002; Jacobson et al., 2008).

The combined evidence of the capabilities of the PN to both modulate its output and interpret its modulated inputs fits the temporal pattern generation theory of cerebellar function. It also suggests that the $\mathrm{PN}$ can itself operate as a pattern/function generator, as opposed to only the IO (Yarom and Cohen, 2002; Rokni et al., 2009) or the granule layer (D'Angelo et al., 2009). By combining variations of the three frequencies, the PN can create any waveform in its output. This can be understood by using the inverse FT, going from right to left in Figure 1A: every signal can be decomposed into a series of harmonics using the FT. This theory of pattern generators is in addition to other theories of cerebellar computation capabilities, including adaptive learning (Raymond et al., 1996; Dean et al., 2010) and control theory (Doya et al., 2001; Ito, 2006), and does not contradict the other theories.

\section{ASTABILITY, BISTABILITY, AND MONOSTABILITY: THE MODES OF A MULTIVIBRATOR}

Currently, there is a controversy in the field regarding the existence of Bistability in the PN output. The disagreement stems from initial reports of "Bistability" in PNs in vitro (Williams et al., 2002), followed by in vitro studies in the presence of anesthetics (Loewenstein et al., 2005). These reports were followed up by a comment paper (Schonewille et al., 2006), which reported that PNs have no Bistability in vivo in awake animals, and only operate in the "upstate." This "up-state" is occasionally broken up by short pauses that are thought to be the major transmitters of PN neuronal information (Jaeger and Bower, 1994; De Schutter and Steuber, 2009; Rokni et al., 2009). The comment paper claimed that Bistability was an artifact of the anesthesia preparation, or the result of "damaged cells." This damaged cell hypothesis has become the de facto currently accepted theory regarding the pauses seen in PN recordings.

It is instructive to give a brief description of these terminologies, from the perspective of EE, from where these terms originated. The term Bistable can only be used for a system that has two characteristics: First, that it has two ("Bi-") states that can remain stable indefinitely without external inputs. Second, an input trigger

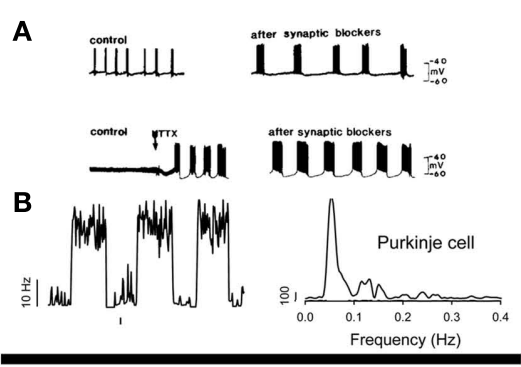

C

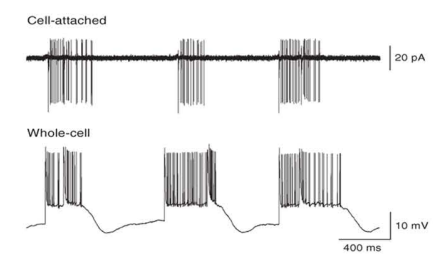

D
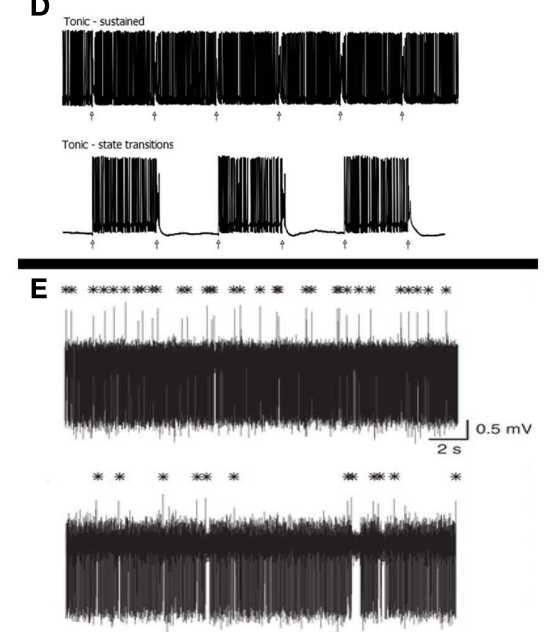

$\mathbf{F}$

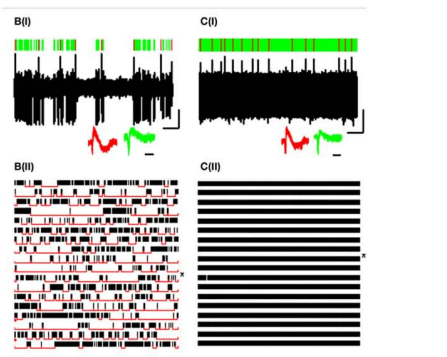

FIGURE 4 | Previous experimental evidence of Astable, Bistable, and Monostable firing in Purkinje neurons. (A) First description of long period $(<1 \mathrm{~Hz})$ bursting in Purkinje recordings in vitro as being an intrinsic characteristic of these cells. Firing pattern was shown to be unaffected by synaptic blockers (Chang et al., 1993). (B) In vivo demonstrations of low-frequency Astable oscillations in the molecular layer of tottering mice measured using fluorescence (Chen et al., 2009). (C) In vitro demonstrations of Bistability consisting of alternating and near-random periods of low-membrane-potential quiescence and high-membrane-potential rapid firing (Loewenstein et al., 2005). (D) Bistability transitions in vitro via the climbing-fiber input to the Purkinje (arrows), acting as a trigger between states (McKay et al., 2007). (E) In vivo demonstration of Purkinje neurons being primarily in the "up-state," with occasional pauses (Schonewille et al., 2006). (F) In vivo demonstration of both Bistability (left, with many long pauses) and Monostability (right, with few to none pauses) in Purkinje recordings (Yartsev et al., 2009). 
to a Bistable system will switch it between the two states, and this trigger can operate in an asynchronous manner. A prime example of a Bistable system is a memory bit, which retains its value indefinitely until erased or rewritten. A Bistable system is inherently connected to two other systems: Astable and Monostable systems. Astable systems have no ("A-") stable states; this means that the system will oscillate if it has two states, but cannot remain at any one state indefinitely. Monostable systems have one ("Mono-") state that can be maintained indefinitely, and an external trigger is needed to transition it between states. However, since the system has only one stable state, the transition will be short, and the system will equilibrate back to its single stable state. These three modalities are characteristic behaviors of multivibrator circuits.

Using this terminology as strictly defined in EE use, we find that the PN has been experimentally shown to have all three modalities, if we define the two states as being the firing state ("up-state"), and the quiescent state. In particular, the "Switching" frequency described by us (Abrams et al., 2010) as occurring in in vitro PN preparations is easily seen to be an Astable mode. This Astable mode has been seen by others in the past in in vitro (Chang et al., 1993) and in vivo (Chen et al., 2009) preparations, as shown in Figures 4A,B, respectively. Unfortunately, since the term "Astability" was apparently unbeknownst to some, the term Bistability has also been used to describe oscillating states (coined "enhanced Bistability"; Williams et al., 2002). This discrepancy, as well as other claims of a lack of oscillatory patterns in the PN (Hakimian et al., 2008), have lead to some inconsistencies in the descriptions.

Bistability has been shown in many systems in vitro, particularly in anesthetized animals, with the trigger found to be the CF signal (Loewenstein et al., 2005; McKay et al., 2007; Davie et al., 2008; Rokni and Yarom, 2009), as shown in Figures 4C,D. The complete modulation functionalities of the CF have not yet been fully determined, with many other theories existing (De Schutter and Steuber, 2009; Ohtsuki et al., 2009). State transitions have also been shown to occur via inhibitory inputs (Oldfield et al., 2010). Monostability is claimed to be the predominant state in awake animals in vitro (Schonewille et al., 2006), as first demonstrated in Figure 4E, however recent work has shown the presence of Bistability in awake animals as well (Yartsev et al., 2009), as shown in Figure 4F. Nevertheless, this latter work has been considered spurious by some, due to its contradiction of the "damaged cell" hypothesis (O. Donchin, personal communication, 2011).

\section{CIRCUIT MODEL OF THE SWITCHING FREQUENCY}

Considering the experimental results of the past decade, we here postulate a relationship between the inherent Switching frequency (Abrams et al., 2010) and the modalities of a multivibrator, with various means of asynchronously modulating this frequency. The Switching frequency can be described as a function of a multivibrator, oscillating (Astable), turning the signal on or off (Bistable), or even creating short "pauses" (Monostable). The term "Switching" was used to describe this frequency range precisely for this reason, as it would explain why the multivibrator modalities are occasionally detected in distinct phases, and can be externally "switched" between modes.

By using the multivibrator circuit analogy, one would expect these three modalities to be present, since they are elementary components of a timing circuit. Figures 5A-F display the three basic timing circuit capabilities of two different multivibrator configurations; the first based on an operational amplifier (Op-Amp; Figures 5A-C), and the second using a 555 chip (Figures 5D-F). The 555 timer circuit and the Op-Amp are probably the two most prevalent chips in existence today (Sedra and Smith, 1998), and are used in applications ranging from complex computers to toys. By merely changing the configuration of the external resistors and capacitors, the three modalities are easily interchanged. One major difference between the modalities is that the Astable circuit requires no inputs, whereas the Monostable and Bistable circuits require external input triggers to modulate between states (orange input triggers in Figure 5). As described above, it is known that the CF input (e.g.) to the PN can act as such a trigger.

The depiction of the 555 timing circuit is instructive in terms of reverse-engineering. The 555 can be seen as a "black box," since its internals are not shown, and yet the function of the overall circuit, including the external passive elements ( $R, C$, etc.), is quite easy to decipher when viewing the output. While the Op-Amp and 555 circuits depicted in Figure 5 perform the same function, the mechanisms to do so are slightly different. Differentiating between the overall function of the circuit, and the internal mechanisms with which they are created is appropriate for deciphering the PN function: while we show that the PN can act as a multivibrator, with an oscillatory function that is similar to the IO's, it may have additional capabilities yet unknown to us.

This connection between the three modalities is also a direct result of the Hodgkin-Huxley equations of a neuron (Hodgkin and Huxley, 1952) that form the basis of dynamic system modeling in neuroscience (Izhikevich, 2006). A simplified version of these equations can be found in the Morris-Lecar model (Morris and Lecar, 1981), which provides a 2-D representation of these equations. Using this model, Bistability was originally modeled, including the $\mathrm{CF}$ as a modulating input trigger (Loewenstein et al., 2005), as shown in the 2-D model of Figure 5H. In fact, the relation between the three modalities was mentioned in the original Morris-Lecar paper, with an external current, I, differentiating between modalities, as a function of two gating parameters, as is distinctly portrayed in Figure 5I.

This circuit model description, as well as the Morris-Lecar dynamic system description, reveals that the "controversy" regarding the existence of Bistability cannot be ascribed only to "damaged cells," but is an intrinsic behavior of the PN, as measured using multiple methods.

\section{CONCLUSION}

Using tools from EE to understand the fundamental processes occurring at the cellular circuit level are essential if we are to understand the ultimate functionality of the cerebellum. The complex mixture of frequencies apparent in the PN output, characterized by its three experimentally discovered frequency ranges (Abrams et al., 2010), as well as the ability of other cells in the cerebellar circuit to decipher these signals, is an entirely different paradigm of understanding information processes in the cerebellum. The combination of analog and digital circuitry to describe the output signal attests to a complexity of information synthesis that 

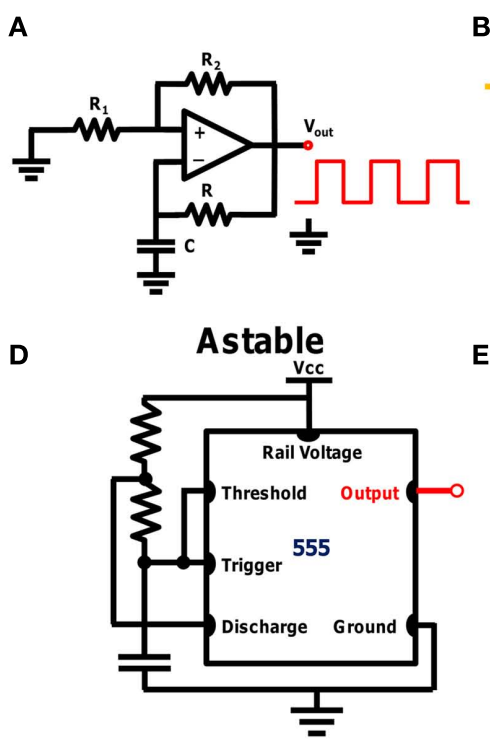

$\mathbf{G}$

H

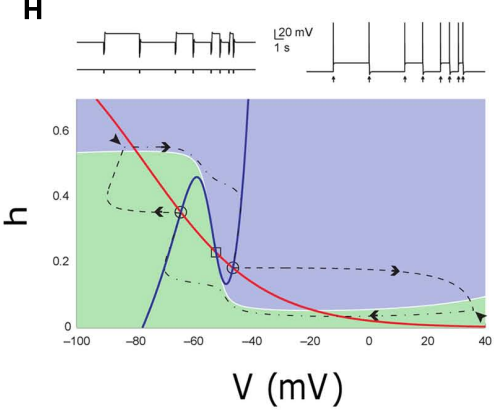

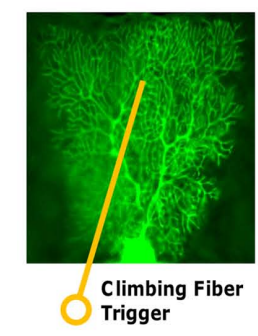

B
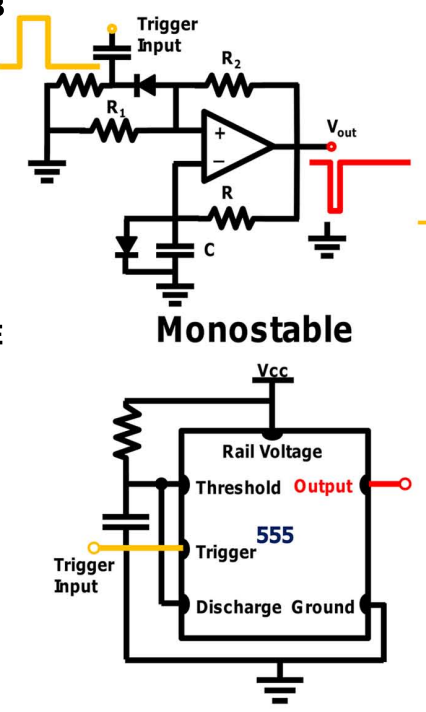

I
C
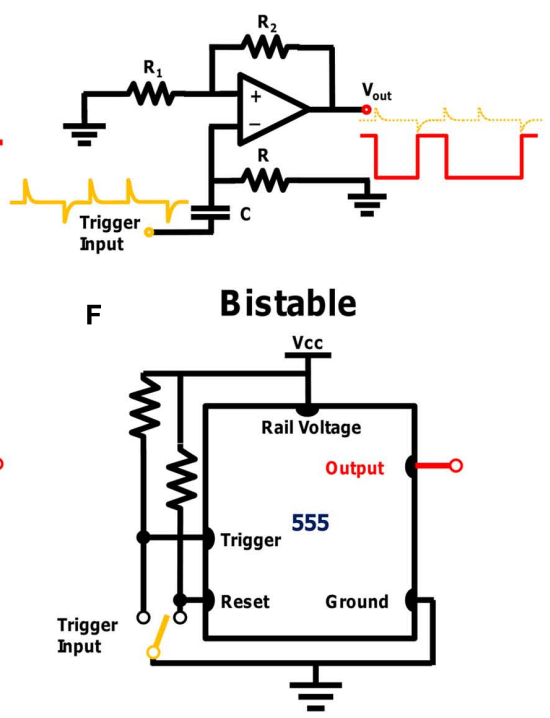

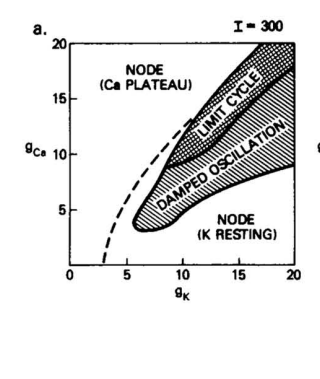

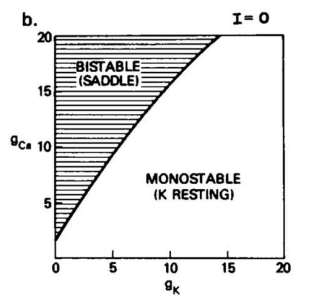

FIGURE 5 | Circuit description of the Bistability controversy. (A-C) display Astable, Monostable, and Bistable circuits (respectively) using an operational amplifier and few passive elements. For each circuit, the bottom RC pair controls the primary timing element: period, duration of Monostability and switching times. The Monostable and Bistable circuits require an external trigger to transition between states (orange), whereas the Astable circuit acts passively as a clock. (D-F): similar circuits using the 555 timer circuit and few passive elements. The ubiquitous 555 circuit's primary usage is to provide these three forms of timing capabilities. (G) Conceptual image of the Purkinje neuron with the climbing-fiber acting as an external trigger, as was demonstrated experimentally by others (see Figure 4). (H) Dynamic systems model of Bistability with an external trigger (Loewenstein et al., 2005). The circles signify the stable states in phase space. External triggers (excitatory or inhibitory) can shift the stability to another state. (I) Original description of a two-parameter Morris-Lecar model system providing all three modes of multivibrator operation as a function of the current and gating variables. The Morris-Lecar model is generalized for any neuronal system (Morris and Lecar, 1981). lies beyond the scope of current analog-to-digital or digital-toanalog converters. Moreover, despite input-output relations such as the linear algorithm shown in Figure 3A (Walter and Khodakhah, 2006), the mixed analog signal of the PN would appear to function beyond the complexity of a basic multiplexor or comparator (Hendry, 2004). Furthermore, the addition of characteristic frequency bands to the firing rate of the PN increases the knowledge of information that the PN can pass to its output (Strong et al., 1998). The ability to modulate the frequencies in an analog and digital manner adds a level of variability to the signal that is beyond the addition of "noise" that is typically described as adding information to the neuronal spike trains (Stein et al., 2005). The signal processing methodology thus provides a new way of viewing the function of the PN on the circuit level.

Finally, using the circuit analysis description of the Switching frequency, we are able to present an alternative theory regarding the current controversy in the field of the cerebellum regarding the absence of Astability/Bistability (Schonewille et al., 2006). This theory fits perfectly into the existing repertoire of literature describing both oscillations (Chen et al., 2009) and Bistable switching (Loewenstein et al., 2005) using a variety of preparation methods and input modalities. However, it relies on reinterpreting the existing measurements of the $\mathrm{PN}$ in terms of multivibrator function and signal analysis, without directly contradicting any of the existing papers in the field.

\section{ACKNOWLEDGMENTS}

Ze'ev R. Abrams was funded with Government support under and awarded by DoD, Air Force Office of Scientific Research, National Defense Science, and Engineering Graduate (NDSEG) Fellowship, 32 CFR 168a. Ze'ev R. Abrams and Xiang Zhang acknowledge support from NSF Nanoscale Science and Engineering Center (SINAM) (CMMI-0751621). 


\section{REFERENCES}

Abrams, Z. R., Warrier, A., Trauner, D., and Zhang, X. (2010). A signal processing analysis of Purkinje cells in vitro. Front. Neural Circuits 4:13. doi: 10.3389/fncir.2010.00013

Achard, P., and De Schutter, E. (2008). Calcium, synaptic plasticity and intrinsic homeostasis in Purkinje neuron models. Front. Compt. Neurosci. 2:8. doi: 10.3389/neuro.10.008.2008

Albus, J. S. (1971). A theory of cerebellar function. Math. Biosci. 10, 25-61.

Apps, R., and Hawkes, R. (2009). Cerebellar cortical organization: a onemap hypothesis. Nat. Rev. Neurosci. 10, 670-681.

Atallah, B., and Scanziani, M. (2009). Instantaneous modulation of gamma oscillation frequency by balancing excitation with inhibition. Neuron 62, 566-577.

Baumel, Y., Jacobson, G. A., and Cohen, D. (2009). Implications of functional anatomy on information processing in the deep cerebellar nuclei. Front. Cell. Neurosci. 3:14. doi: 10.3389/neuro.03.014.2009

Bower, J. M. (2010). Model-founded explorations of the roles of molecular layer inhibition in regulating Purkinje cell responses in cerebellar cortex: more trouble for the beam hypothesis. Front. Cell. Neurosci. 4:27. doi: 10.3389/fncel.2010. 00027

Brunel, N., Hakim, V., Isope, P., Nadal, J. P., and Barbour, B. (2004). Optimal information storage and the distribution of synaptic weights: perceptron versus Purkinje cell. Neuron 43, 745-757.

Buzsáki, G., and Draguhn, A. (2004). Neuronal oscillations in cortical networks. Science 304, 1926-1929.

Chang, W., Strahlendorf, J. C., and Strahlendorf, H. K. (1993). Ionic contributions to the oscillatory firing activity of rat Purkinje cells in vitro. Brain Res. 614, 335-341.

Chen, G., Popa, L. S., Wang, X., Gao, W., Barnes, J., Hendrix, C. M., Hess, E. J., and Ebner, T. J. (2009). Lowfrequency oscillations in the cerebellar cortex of the tottering mouse. J. Neurophysiol. 101, 234-245.

Colgin, L. L., Dennunger, T., Fyhn, M., Hafting, T., Bonnevie, T., Jensen, O., Moser, M. B., and Moser, E. I. (2009). Frequency of gamma oscillations routes flow of information in the hippocampus. Nature 462, 353-357.

D’Angelo, E., Koekkoek, K. E., Lombardo, P., Solinas, S., Ros, E., Garrido, J., Schonewille, M., and De
Zeeuw, C. I. (2009). Timing in the cerebellum: oscillations and resonance in the granular layer. Neuroscience $162,805-815$.

Davie, J. T., Clark, B. A., and Häusser, M. (2008). The origin of the complex spike in cerebellar Purkinje cells. J. Neurosci. 28, 7599-7609.

De Schutter, E., and Steuber, V. (2009). Patterns and pauses in Purkinje cell simple spike trains: experiments, modelling and theory. Neuroscience 162, 816-826.

De Zeeuw, C. I., Hoebeek, F. E., and Schonewille, M. (2008). Causes and consequences of oscillations in the cerebellar cortex. Neuron 58, 655-658.

Dean, P., Porrill, J., Ekerot, C. F., and Jörntell, H. (2010). The cerebellar microcircuit as an adaptive filter: experimental and computational evidence. Nat. Rev. Neurosci. 11, 30-43.

Doya, K., Kimura, H., and Kawato, M. (2001). Neural mechanisms of learning and control. IEEE Control Syst. Mag. 21, 42-54.

Eccles, J. C., Ito, M., and Szentágothai, J. (1967). The Cerebellum as a Neuronal Machine. Berlin: SpringerVerlag.

Ekerot, C. F., and Kano, M. (1985).Longterm depression of parallel fibre synapses following stimulation of climbing fibres. Brain Res. 342, 357-360.

Geisler, C., Diba, K., Pastalkova, E., Mizuseki, K., Royer, S., and Buzsáki, G. (2010). Temporal delays among place cells determine the frequency of population theta oscillations in the hippocampus. Proc. Natl. Acad. Sci. U.S.A. 107, 7957-7962.

Hakimian, S., Norris, S. A., Greger, B., Keating, J. G., Anderson, C. H., and Thach, W. T. (2008). Time and frequency characteristics of Purkinje cell complex spikes in the awake monkey performing a nonperiodic task. J. Neurophysiol. 100, 1032-1040.

Hansel, C., and Linden, D. J. (2000). Long-term depression of the cerebellar climbing fiber-Purkinje neuron synapse. Neuron 26, 473-482.

Hartmann, J., and Konnerth, A. (2005). Determinants of postsynaptic $\mathrm{Ca}_{2}{ }^{+}$ signalling in Purkinje neurons. Cell Calcium 37, 459-466.

Häusser, M., and Clark, B. A. (1997). Tonic synaptic inhibition modulates neuronal output pattern and spatiotemporal synaptic integration. Neuron 19, 665-678.

Hendry, D. C. (2004). Comparator trees for winner-take-all circuits. Neurocomputing 62, 389-403.
Hodgkin, A. L., and Huxley, A. F. (1952). A quantitative description of membrane current and its application to conduction and excitation in nerve. J. Physiol. 117, 500-544.

Isope, P., and Barbour, B. (2002). Properties of unitary granule cell Purkinje cell synapses in adult rat cerebellar slices. J. Neurosci. 22, 9668-9678.

Ito, M. (2001). Cerebellar long-term depression: characterization, signal transduction, and functional roles. Physiol. Rev. 81, 1143-1195.

Ito, M. (2006). Cerebellar circuitry as a neuronal machine. Prog. Neurobiol. 78, 272-303.

Izhikevich, E. M. (2006). Dynamical Systems in Neuroscience: The Geome try of Excitability and Bursting. Cambridge: MIT Press.

Jacobson, G. A., Lev, I., Yarom, Y., and Cohen, D. (2009). Invariant phase structure of olivo-cerebellar oscillations and its putative role in temporal pattern generation. Proc. Natl. Acad. Sci. U.S.A. 106, 3579-3584.

Jacobson, G. A., Rokni, D., and Yarom Y. (2008). A model of the olivocerebellar system as a temporal pattern generator. Trends Neurosci. 31, 617-625.

Jaeger, D., and Bower, J. M. (1994). Prolonged responses in rat cerebellar Purkinje cells following activation of the granule cell layer: and intracellular in vitro and in vivo investigation. Exp. Brain Res. 100, 200-214.

Kano, M., and Kato, M. (1987) Quisqualate receptors are specifically involves in cerebellar synaptic plasticity. Nature 325, 276-279.

Keating, J. G., and Thach, W. T. (1997). No clock signal in the discharge of neurons in the deep cerebellar nuclei. J. Neurophysiol. 77, 2232-2234.

Kramer, M. A., Traub, R. D., and Kopell N. J. (2008). New dynamics in cerebellar Purkinje cells: torus canards. Phys. Rev. Lett. 101, 068103-068106.

Lang, E. J., Sugihara, I., Welsh, J. P., and Llinás, R. (1999). Patterns of spontaneous Purkinje cell complex spike activity in awake rat. J. Neurosci. 18 , 2728-2738.

Llinás, R., and Sugimori, M. (1980). Electrophysiological properties of in vitro Purkinje cell dendrites in mammalian cerebellar slices. J. Physiol. 305, 197-213.

Loewenstein, Y., Mahon, S., Chadderton, P., Kitamura, K., Sompolinsky, H., Yarom, Y., and Häusser, M. (2005). Bistability of cerebellar Purkinje cells modulated by sensory stimulation. Nat. Neurosci. 8 , 202-211.
Maex, R., and De Schutter, E. (2005) Oscillations in the cerebellar cortex: a prediction of their frequency bands. Prog. Brain Res. 148, 181-188.

Mapelli, J., Gandolfi, D., and D'Angelo, E. (2010). High-pass filtering and dynamic gain regulation enhance vertical bursts transmission along the mossy fiber pathway of cerebellum. Front. Cell. Neurosci. 4:14. doi: 10.3389/fncel.2010.00014

Marr, D. (1969). A theory of cerebellar cortex. J. Physiol. 202, 437-470.

Mathy, A., Ho, S. S. N., Davie, J. T., Duguid, I. C., Clark, B. A., and Häusser, M. (2009). Encoding of oscillations by axonal bursts in inferior olive neurons. Neuron 62 , 388-399.

McKay, B. E., Engbers, J. D. T., Mehaffey, W. H., Gordon, G. R. J., Molineux, M. L., Bains, J. S., and Turner, R. W. (2007). Climbing fiber discharge regulates cerebellar functions by controlling the intrinsic characteristics of Purkinje cell output. J. Neurophysiol. 97, 2590-2604.

Mittmann, W., and Häusser, M. (2007). Linking synaptic plasticity and spike output at excitatory and inhibitory synapses onto cerebellar Purkinje cells. J. Neurosci. 27, 5559-5570.

Monsivais, P., Clark, B. A., Roth, A., and Häusser, M. (2005). Determinants of action potential propagation in cerebellar Purkinje cell axons. J. Neurosci. 25, 464-472.

Morris, C., and Lecar, H. (1981). Voltage oscillations in the barnacle giant muscle fiber. Biophys. J. 35, 193-213.

Ohtsuki, G., Piochon, C., and Hansel, C. (2009). Climbing fiber signaling and cerebellar gain control. Front. Cell. Neurosci. 3:4. doi: 10.3389/neuro.03.004.2009

Oldfield, C. S., Marty, A., and Stell, B. M. (2010). Interneurons of the cerebellar cortex toggle Purkinje cells between up and down states. Proc. Natl Acad. Sci. U.S.A. 107, 13153-13158.

Raymond, J. L., Lisberger, S. G., and Mauk, M. D. (1996). The cerebellum: a neuronal learning machine? Science 272, 1126-1131.

Rokni, D., Llinás, R., and Yarom, Y. (2008). The morpho/functional discrepancy in the cerebellar cortex: looks alone are deceptive. Front. Neurosci. 2:36. doi: 10.3389/neuro.01.036.2008.

Rokni, D., Tal, Z., Byk, H., and Yarom, Y. (2009). Regularity, variability and bi-stability in the activity of cerebellar Purkinje cells. Front. Cell. Neurosci. 3:12. doi: 10.3389/neuro.03.012.2009 
Rokni, D., and Yarom, Y. (2009). State-dependence of climbing fiber-driven calcium transients in Purkinje cells. Neuroscience 162, 694-701.

Roopun, A. K., Kramer, M. A., Carracedo, L. M., Kaiser, M., Davies, C. H., Traub, R. D., Kopell, N. J., and Whittington, M. A. (2008). Temporal interactions between cortical rhythms. Front. Neurosci. 2:34. doi: 10.3389/neuro.01.034. 2008

Schonewille, M., Khosrovani, S., Winkelman, B. H. J., De Jeu, M. T. G., Larsen, I. M., Van Der Burg, J., Schmolesky, M. T., Frens, M. A., and De Zeeuw, C. I. (2006). Purkinje cells in awake behaving animals operate at the upstate membrane potential. Nat. Neurosci. 9, 459-461.

Sedra, A. S., and Smith, K. C. (1998). Microelectronic Circuits. New York: Oxford University Press.
Stein, R. B., Gossen, E. R., and Jones, K. E. (2005). Neuronal variability: noise or part of the signal? Nat. Rev. Neurosci. 6, 389-397.

Steriade, M. (2006). Grouping of brain rhythms in corticothalamic systems. Neuroscience 137, 1087-1106.

Strong, S. P., Koberle, R., de Ruyter van Steveninck, R. R., and Bialek, W. (1998). Entropy and information in neural spike trains. Phys. Rev. Lett. 80, 197-200.

Telgkamp, P., and Raman, I. M. (2002). Depression of inhibitory synaptic transmission between Purkinje cells and neurons of the cerebellar nuclei. J. Neurosci. 22, 8447-8457.

Walter, J. T., and Khodakhah, K. (2006). The linear computational algorithm of cerebellar Purkinje cells. J. Neurosci. 26, 12861-12872.

Williams, R. S., Christensen, S. R., Stuart, G. J., and Haüsser, M. (2002). Membrane potential bistability is controlled by the hyperpolarizationactivated current Ih in rat cerebellar
Purkinje neurons in vitro. J. Physiol. 539, 469-483.

Wise, A. K., Cerminara, N. L., MarpleHorvat, D. E., and Apps, R. (2010). Mechanisms of synchronous activity in cerebellar Purkinje cells. J. Physiol. 588, 2373-2390.

Womack, M., and Khodakhah, K. (2002). Active contribution of dendrites to the tonic and trimodal patterns of activity in cerebellar Purkinje neurons. J. Neurosci. 15, 10603-10612.

Womack, M., and Khodakhah, K. (2004). Dendritic control of spontaneous bursting in cerebellar Purkinje cells. J. Neurosci. 24, 3511-3521.

Yarom, Y., and Cohen, D. (2002). The olivocerebellar system as a generator of temporal patterns. Ann. N. Y. Acad. Sci. 978, 122-134.

Yartsev, M. M., Givon-Mayo, R., Maller, M., and Donchin, O. (2009). Pausing Purkinje cells in the cerebellum of the awake cat. Front. Sys. Neurosci. 3:2. doi: 10.3389/neuro.06.002. 2009

Conflict of Interest Statement: The authors declare that the research was conducted in the absence of any commercial or financial relationships that could be construed as a potential conflict of interest.

Received: 12 July 2011; accepted: 05 September 2011; published online: 26 September 2011.

Citation: Abrams $Z R$ and Zhang $X$ (2011) Signals and circuits in the Purkinje neuron. Front. Neural Circuits 5:11. doi: 10.3389/fncir.2011.00011

Copyright (c) 2011 Abrams and Zhang. This is an open-access article subject to a non-exclusive license between the authors and Frontiers Media SA, which permits use, distribution and reproduction in other forums, provided the original authors and source are credited and other Frontiers conditions are complied with. 